\title{
$A b$ initio molecular dynamics simulations of low energy recoil events in MgO
}

\author{
B. A. Petersen ${ }^{1}$, B. Liu ${ }^{1}$, W. J. Weber ${ }^{1,2, *}$ and Y. Zhang ${ }^{2,1, *}$ \\ ${ }^{1}$ Department of Materials Science and Engineering, University of Tennessee, Knoxville, Tennessee \\ 37996, USA \\ ${ }^{2}$ Materials Science and Technology Division, Oak Ridge National Laboratory, Oak Ridge, Tennessee \\ 37831, USA \\ *E-mail: wjweber@utk.edu and zhangy1@ornl.gov
}

\begin{abstract}
Low-energy recoil events in $\mathrm{MgO}$ are studied using ab intio molecular dynamics simulations to reveal the dynamic displacement processes and final defect configurations. Threshold displacement energies, $E_{\mathrm{d}}$, are obtained for $\mathrm{Mg}$ and $\mathrm{O}$ along three low-index crystallographic directions, [100], [110], and [111]. The minimum values for $E_{\mathrm{d}}$ are found along the [110] direction consisting of the same element, either $\mathrm{Mg}$ or $\mathrm{O}$ atoms. Minimum threshold values of $29.5 \mathrm{eV}$ for $\mathrm{Mg}$ and $25.5 \mathrm{eV}$ for $\mathrm{O}$, respectively, are suggested from the calculations. For other directions, the threshold energies are considerably higher, 65.5 and 150.0 $\mathrm{eV}$ for $\mathrm{O}$ along [111] and [100], and $122.5 \mathrm{eV}$ for $\mathrm{Mg}$ along both [111] and [100] directions, respectively. These results show that the recoil events in $\mathrm{MgO}$ are partial-charge transfer assisted processes where the charge transfer plays an important role. There is a similar trend found in other oxide materials, where the threshold displacement energy correlates linearly with the peak partial-charge transfer, suggesting this behavior might be universal in ceramic oxides.
\end{abstract}

\section{Introduction}

Advancement in reliable prediction of formation and evolution of radiation-induced defects has garnered renewed interest in the realm of ion-solid interaction, which is essential to evaluate material performance to radiation in various applications, such as space exploration, nuclear power plants and nuclear waste disposal, as well as high-energy accelerators. Of particular importance is investigating the material response in radiation environment in terms of microstructural rearrangement and evolution (i.e. defect production, phase transitions, atomic diffusion, etc.). Several ceramic oxides are identified as candidates for use as waste forms for the long term storage of transuranium elements or actinide transmutation targets due to their chemical and physical stability under irradiation conditions $[1,2]$. 
Magnesium oxide, $\mathrm{MgO}$, has been extensively studied, both experimentally and theoretically, due to its usefulness as an engineering ceramic and as a model ceramic material for nuclear energy applications [3]. In nuclear energy applications, $\mathrm{MgO}$ has been investigated as an inert matrix for nuclear fuels [4], as an insulator for fusion reactor designs, and as a useful material for radioactive waste disposal $[1,5]$. As a transmutation target, $\mathrm{MgO}$ is a promising candidate due to its low neutron cross section and high thermal conductivity in addition to its radiation resistance. The simple cubic crystal structure and the associated electronic and atomic arrangement of $\mathrm{MgO}$ suggest it can be considered as a model material to investigate radiation effects and defect mobility in ceramic oxides.

In nuclear environments, radiation events, such as decay of radio-nuclides or elastic collisions of neutrons with atomic nuclei, produce energetic charged particles. The interaction of target atoms with these energetic particles can set many atoms in motion and creates localized disorder in the lattice [6]. In most ceramic materials under irradiation, atomic displacement events can be predicted by the KinchinPease model and codes like Transport of Ions in Matter (TRIM which is part of the SRIM (Stopping and Range of Ions in Matter) software package) [7]. These atomic displacements create numerous point defects, most of which undergo simultaneous recombination, leaving a few stable defects behind. Accumulation of immobile defects may result in amorphization, while migration and recombination of mobile defects at higher temperatures can lead to microstructural modification. The experimental defect production rate under irradiation is determined by the local rate of atomic displacement survival, which results from the competition between defect production and instantaneous recombination during collision cascade events, and this is typically described as a dose rate and given in displacements per atom per second $\left(\mathrm{dpa}-\mathrm{s}^{-1}\right)$.

Key knowledge in evaluating a specific material's resistance to interactions with fission fragments, other byproducts of nuclear decay and neutron collisions is the description of low-energy events, where understanding the dynamic displacement processes and defect configurations at the end of the collision cascade process is essential. In low-energy events, the threshold displacement energy, $E_{\mathrm{d}}$, is defined as the kinetic energy needed to displace an atom, and the energetic particle (one of the atoms on the lattice given a specific kinetic energy), known as a primary knock-on atom (PKA), may be permanently off its initial lattice site to form a stable defect. The definition of threshold displacement energy in this work is the minimum kinetic energy an atom in the solid needs to be displaced from its lattice site to a defect site or substitutional position on the lattice [6]. This threshold displacement energy value, or an average value, can be used in various models to estimate the total number of displacements per radiation event or per incident ion, the depth distribution of damage for external charged particle irradiations, and the effective dose rate. Many models, such as the Kinchin-Pease model or codes like TRIM that rely on a Binary Collision Approximation (BCA), require the average $E_{\mathrm{d}}\left(E_{\mathrm{d}}\right.$ ave $)$ summed 
across many lattice directions to properly estimate displacement events in materials $[6,8]$. Compared with the Frenkel pair formation energy, $E_{\mathrm{d}}$ is higher due to the multibody, non-equilibrium kinetics involved [8]; determining $E_{d}$ therefore requires a more computationally expensive procedure.

Experimentally, $E_{\mathrm{d}}$ is difficult to measure due to the short time scales involved, the lack of facilities, and close dependence on direction and sublattice orientation (imperfect alignment of incident ions or electrons respect to the crystallographic direction due to experimental error), as well as the methods used to quantify defects and displacement events [6]. Simulations are used to determine $E_{\mathrm{d}}$. Radiation damage simulations based on classical molecular dynamics (MD) have been utilized to produce reasonable results [9-12]. Classical models, however, neglect many important effects, such as the charge effect due to electron redistribution during collision cascades that has been found to play an important role in defect production and migration in ceramic materials [13,14]. In order to address this deficiency and to provide insight into the fundamental mechanisms of defect production and nature of defects produced, ab initio molecular dynamics (AIMD) simulations are used in this study. The low-energy recoil events for both the $\mathrm{Mg}$ and $\mathrm{O}$ sublattices in $\mathrm{MgO}$ are investigated along three low index crystallographic directions, [100], [110], and [111]. Determining the average $E_{d}$ value requires averaging over all possible lattice directions and can be very computational expensive for AIMD simulations due to the long distances that recoil atoms can travel, as well as the computational time constraints. By limiting the recoils to low index directions, they are more likely to interact immediately with surrounding atoms to provide a general description of the production and configuration of defects under irradiation [8]. The anisotropic threshold displacement energies are determined, together with the associated defect configurations and the mechanisms for defect formation. Partial-charge transfer and its effect on the threshold displacement energies are also revealed. Our results are compared with literature results from classical MD simulations and experiments.

\section{Computational details}

All of the AIMD simulations were performed using a modified version of the SIESTA code $[14,15]$. Interactions between atoms and electrons were described by norm-conserving Troullier-Martins psuedopotentials [16] factorized in the Kleinman-Bylander form [17]. The exchange correlation functional used was determined within the generalized-gradient approximation (GGA) as parameterized by Perdew, Burke, and Ernzerhof (PBE) [18]. The reference electronic configurations used were $3 \mathrm{~s}^{2}$ and $2 \mathrm{~s}^{2} 2 \mathrm{p}^{4}$ for $\mathrm{Mg}$ and $\mathrm{O}$, respectively. The $\mathrm{Mg}$ pseudopotential core radii (Bohr) and occupation are 2.18 and 2.00 for $3 \mathrm{~s}$ electrons, and 2.59 and 0.00 for $3 \mathrm{p}, 3 \mathrm{~d}$ and $4 \mathrm{f}$ electrons, respectively. The $\mathrm{O}$ pseudopotential core radius (Bohr) is 1.47 for $3 \mathrm{~s}, 3 \mathrm{p}, 3 \mathrm{~d}$ and $4 \mathrm{f}$ electrons, and the occupation is 2.00 and 4.00 for $3 \mathrm{~s}$ and $3 p$ electrons but 0.00 for $3 \mathrm{~d}$ and $4 \mathrm{f}$ electrons, respectively. The cutoff energy was set to $70 \mathrm{Ry}$ for the 
basis set. K-point sampling of $1 \times 1 \times 1$ in the Brillouin zone was used. Valance wave functions were expanded in the basis set of localized atomic orbitals. Double- $\zeta$ basis sets with polarization orbitals were used.

For all simulations, the NVE ensemble was used. Initially, simulations were carried out in a $3 \times 3$ $\times 3$ unit cell containing 216 atoms. Due to the use of periodic boundary conditions (PBCs), the recoil event must be fully contained within the supercell or else it has the potential to interact with itself. To prevent the recoil from interfering with itself, various cell sizes were selected: $3 \times 3 \times 3$ (216 atoms) for O [110] and $\mathrm{O}$ [111]; $4 \times 3 \times 3$ (288 atoms) for $\mathrm{Mg}$ [100] and $\mathrm{O}$ [100]; and $4 \times 4 \times 3$ (384 atoms) for $\mathrm{Mg}$ [110] and $\mathrm{Mg}$ [111]. Increasing the cell size does not significantly affect the results, similar to previous studies [19-21]. The initial temperature of the system was set to $0 \mathrm{~K}$ in order to eliminate the influence of random velocities; increasing the temperature of AIMD in $\mathrm{E}_{\mathrm{d}}$ simulations has been shown to increase the value of $E_{\mathrm{d}}$ [20]. Consequently, AIMD simulations for $E_{\mathrm{d}}$ or other low energy recoil events are frequently performed at $0 \mathrm{~K}$ [19-21], if the ground state structure is stable at that temperature. For all simulations, a fixed time step of $1 \mathrm{fs}$ was used. To verify the validity of this choice, a Mg PKA along the [100] direction was repeated for a time step of $1 \mathrm{fs}$ and $0.5 \mathrm{fs}$. Results for the threshold displacement energy and final defect configurations for both time steps did not vary. A maximum time duration of $600 \mathrm{fs}$ proved sufficient, since no any further development of defect structure could be observed with longer computing time, as was done for $\mathrm{Mg}$ and $\mathrm{O}$ PKAs along the [100] direction.

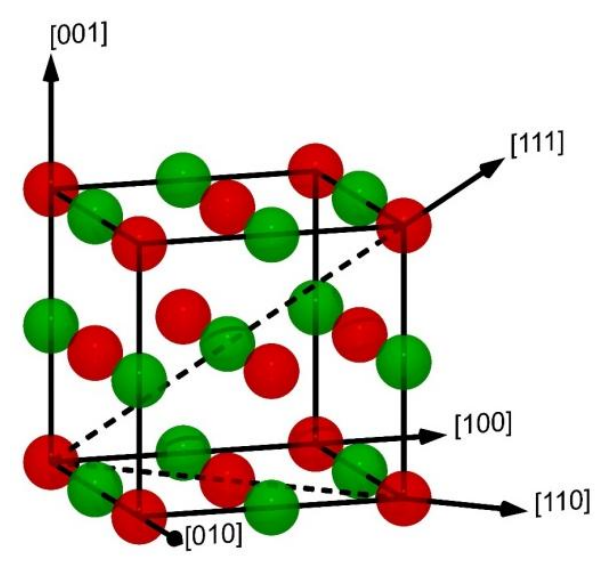

Figure 1: MgO unit cell with three crystallographic indexes, [100], [110], and [111]. Red atoms are O and green atoms are $\mathrm{Mg}$.

Each recoil event was initiated by a PKA with a specific energy and direction. Three low index directions were studied, [100], [110] and [111]. As illustrated in figure 1, every direction in the $\langle 100\rangle$ family is equivalent to the [100] direction. This is the same for all families of directions tested. Initially $E_{\mathrm{d}}$ was set to $60 \mathrm{eV}$ for all cases based on the summary paper from Zinkle and Kinoshita in 1997 that 
suggests values of $55 \mathrm{eV}$ for both $\mathrm{Mg}$ and $\mathrm{O}$ for use with codes like TRIM [6]. If the chosen energy does not displace the PKA off of its initial lattice site, the energy is increased by an increment of $10 \mathrm{eV}$ until it has become displaced. If the PKA is displaced by the initial given energy, a new PKA simulation is then carried out with the energy reduced by an increment of $10 \mathrm{eV}$. Values were then refined as necessary by either increasing or decreasing the PKA energy within a tolerance of $0.5 \mathrm{eV}$.

\section{Results}

\subsection{Ground state properties}

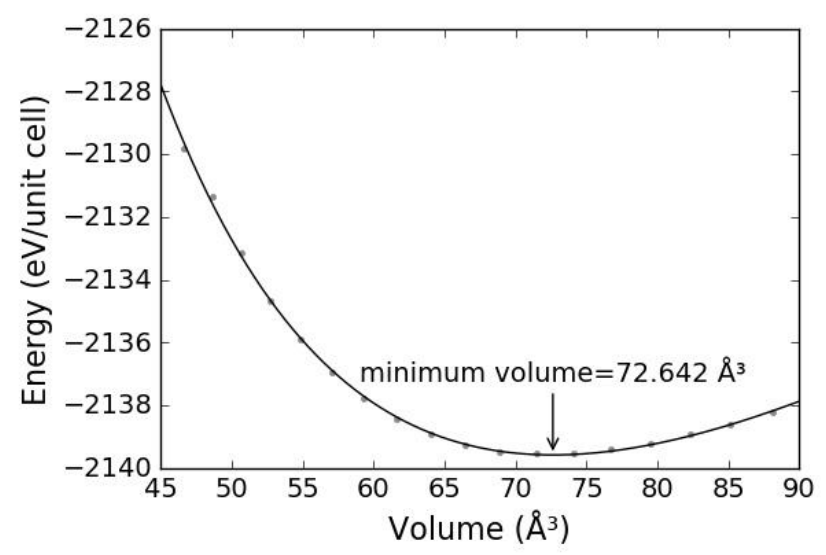

Figure 2: Energy of the $\mathrm{MgO}$ unit cell versus volume. The curve is given by fitting to the BirchMurnaghan equation of state. The arrow shows the point that represents the minimum volume calculated from the fit shown by the curve.

The result in Figure 2 shows the variation in energy of the system versus volume of the unit cell. When varying the volume of a simulation cell but constraining the lattice sites, fitting the energy versus volume to an equation of state relates yields the lattice parameter at the minimum energy and the associated bulk modulus. A least-squares fit to the Birch-Murnaghan equation of state yields a lattice parameter $a$ of $4.172 \AA$ and a bulk modulus $B$ of $182 \mathrm{GPa}$ at the minimum energy of the curve in figure 2 . The calculated value of $a$ agrees well with experimental values ranging from 4.210 to $4.220 \AA$ [22-26]; $B$ is in fair agreement with experimental values of 163.9 [27], 162.20 [28], and $156 \mathrm{GPa}$ [29], and is also consistent with the values from DFT calculations of 172 [30], 185.9, and 169.1 GPa [31].

\subsection{Threshold displacement energies}

The results for calculated $E_{\mathrm{d}}$ values are summarized in table 1 and figure 3 . The values were determined by whether or not the PKA remained displaced from its original lattice position on the 
simulation time scale, while adjusting the kinetic energy of the PKA, as in the procedure described above. Once the simulation had run long enough that the displaced atoms remained in a stable configuration, the defect structure could then be analyzed. The distance traveled by the PKA by the end of the simulation and the resulting defect configuration are given in table 1.

Table 1: Recoil direction, threshold displacement $\left(E_{\mathrm{d}}\right)$, distance the PKA traveled $\left(d_{\mathrm{PKA}}\right)$, and the final defect state for each PKA. PKAs are indicated by (PKA).

\begin{tabular}{ccrrc}
\hline PKA & Direction & $E_{\mathrm{d}}(\mathrm{eV})$ & \multicolumn{1}{c}{$d_{\text {pka }}(\AA)$} & Defect state \\
\hline \multirow{3}{*}{$\mathrm{Mg}$} & {$[100]$} & 122.5 & 4.22 & $\mathrm{Mg}(\mathrm{PKA})_{\mathrm{Mg}}+\mathrm{V}_{\mathrm{O}}+\mathrm{V}_{\mathrm{Mg}}+\mathrm{O}_{\mathrm{i}}+\mathrm{Mg}_{\mathrm{i}}$ \\
& {$[110]$} & 29.5 & 2.95 & $\mathrm{Mg}(\mathrm{PKA})_{\mathrm{Mg}}+\mathrm{Mg}_{\mathrm{Mg}}+\mathrm{V}_{\mathrm{Mg}}+\mathrm{Mg}_{\mathrm{i}}$ \\
& {$[111]$} & 122.5 & 5.11 & $\mathrm{Mg}(\mathrm{PKA})_{\mathrm{Mg}}+\mathrm{Mg}_{\mathrm{Mg}}+\mathrm{Mg}_{\mathrm{Mg}}+\mathrm{Mg}_{\mathrm{Mg}}+\mathrm{O}_{\mathrm{O}}+\mathrm{O}_{\mathrm{O}}+\mathrm{V}_{\mathrm{O}}+\mathrm{V}_{\mathrm{Mg}}+\mathrm{O}_{\mathrm{i}}+\mathrm{Mg}_{\mathrm{i}}$ \\
& {$[100]$} & 150 & 1.48 & $\mathrm{~V}_{\mathrm{O}}+\mathrm{V}_{\mathrm{O}}+\mathrm{O}(\mathrm{PKA})_{\mathrm{i}}+\mathrm{O}_{\mathrm{i}}$ \\
$\mathrm{O}$ & {$[110]$} & 25.5 & 2.36 & $\mathrm{~V}_{\mathrm{O}}+\mathrm{O}(\mathrm{PKA})_{\mathrm{i}}$ \\
& {$[111]$} & 65.5 & 3.76 & $\mathrm{~V}_{\mathrm{O}}+\mathrm{O}(\mathrm{PKA})_{\mathrm{i}}$ \\
\end{tabular}

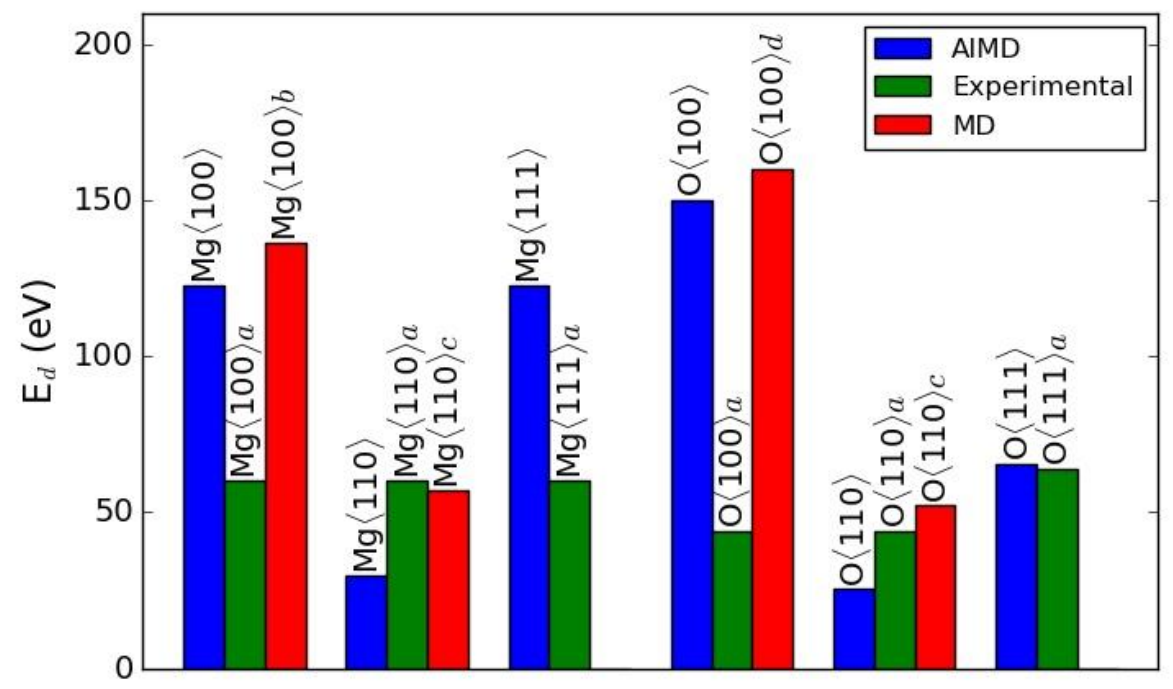

Figure 3: Comparison of $\mathrm{E}_{\mathrm{d}}$ between this work and other studies. Experimental values, $a$, were taken from Pells [32]. Classical MD simulations $b, c$, and $d$, were taken from references [33], [34], and [35], respectively. 

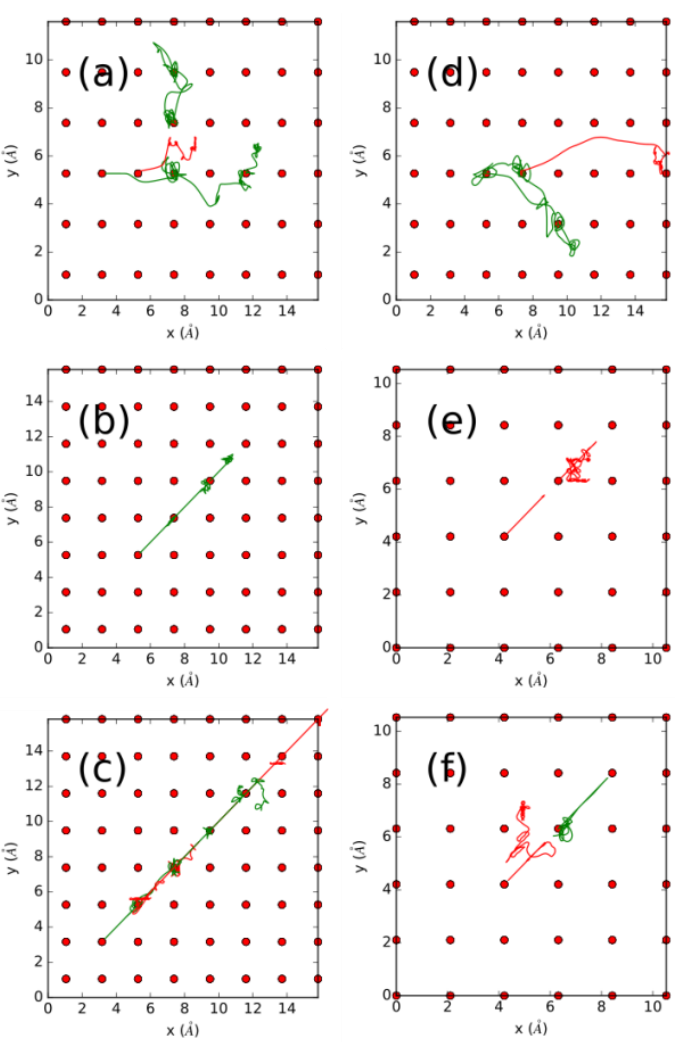

Figure 4: (a) through (c) show traces over the course of a set of simulations of $\mathrm{Mg}[100], \mathrm{Mg}[110]$ and $\mathrm{Mg}[111]$ PKAs projected along the x-y plane. Plots from (d) to (f) show the same for the set of O[100], $\mathrm{O}[110]$, and $\mathrm{O}[111]$ PKAs. Traces shown are atoms that move significantly from their original lattice positions. Red traces are $\mathrm{O}$ atoms and green traces are $\mathrm{Mg}$ atoms. Red dots show the lattice sites of $\mathrm{Mg}$ and $\mathrm{O}$ for reference.

Once an atom has begun moving during the simulations, it generally maintains its path along the initial direction. Some of the recoils, like $\mathrm{Mg}$ [111], gradually change direction (i.e., scatter) during the simulation, eventually aligning with a $\langle 110\rangle$ direction. The traces shown in figure 4 of the PKAs and atoms that move significantly during the simulation show this tendency to form displacements along the $\langle 110\rangle$ directions. During all of the simulations, two primary interstitial defects evolved independently of the PKA species. As shown in figure 5(a), the Mg interstitial sits at a cube-centered site, with the cube being made of $\mathrm{O}$ and $\mathrm{Mg}$ ions. The $\mathrm{O}$ interstitial occupies a split interstitial configuration, shown in figure 5(b). The split O interstitials are suggested from theoretical calculations; DFT and classical molecular dynamics calculations have shown that these split $\mathrm{O}$ interstitials align along either a $\langle 110\rangle$ or $\langle 111\rangle$ direction, depending on the charge state of the defect [13,36,37]. All of the O defects observed during these simulations, however, aligned with a $\langle 110\rangle$ direction. Compared to DFT calculations, this configuration agrees with results produced by neutrally charged $\mathrm{O}$ interstitials $[36,37]$. 


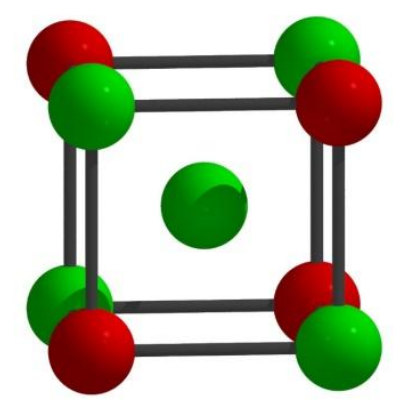

(a)

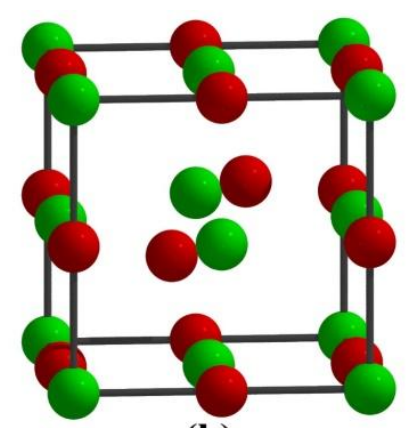

(b)

Figure 5: Interstitial configurations for $\mathrm{Mg}$ and $\mathrm{O}$. (a) shows $\mathrm{Mg}$ (green) at a cube centered site and (b) shows $\mathrm{O}$ (red) as a split interstitial oriented along [110].

There are two main types of interactions for a lattice site during the simulation - either the PKA replaces a lattice atom, which can go on to create one or more defects, or it forms one or more defects beyond the nearest neighbor atom. Figure 6 shows the total number of each type of interaction for all of the PKAs. As detailed as the final defect states in table 1, a low-energy PKA in this study produces at most two interstitials that remained stable at the end of the simulation. No simulation produced more than one $\mathrm{Mg}$ interstitial, and those that produced two interstitials ( $\mathrm{Mg}$ [100], $\mathrm{Mg}$ [111], and $\mathrm{O}$ [100]) produced either two O Frenkel pairs (O [100]) or one Mg and one O Frenkel pair. None of the O PKAs produced any stable O replacements, but the $\mathrm{Mg}$ [111] recoil produced four stable $\mathrm{Mg}$ replacements.

During the Mg displacement event initiated along [100], the primary chain of displacements and replacements is along the initial [100] direction demonstrated in figure 4(a). The Mg PKA displaces the next atom $(\mathrm{O})$ in the chain of atoms along [100]. That displaced $\mathrm{O}$ then forms an interstitial, leaving an $\mathrm{O}$ vacancy behind. Before finally coming to rest in a stable configuration, the displaced $\mathrm{O}$ atom displaces 3 $\mathrm{Mg}$ atoms along [011] immediately adjacent to it, but these three $\mathrm{Mg}$ relax back to their initial lattice sites during the simulation. The PKA then replaces the next atom $(\mathrm{Mg})$ along the [100] direction and remains on that lattice site. The transferred energy is still high enough to push the next oxygen atom in the chain to displace the $\mathrm{Mg}$ atom adjacent to it along the [100] direction. The oxygen atom also causes some temporary displacements of $\mathrm{Mg}$ atoms in the [011] direction, but those atoms return to their original lattice sites near the end of the simulation. In total, two Frenkel pairs (1 Mg and $1 \mathrm{O})$ are formed, and the PKA has replaced the next $\mathrm{Mg}$ atom along [100].

Three $\mathrm{Mg}$ atoms in total are displaced during threshold displacement simulation for the $\mathrm{Mg} \mathrm{PKA}$ along the [110] direction in figure 4(b). The first two events along [110] are replacements. The PKA replaces the first neighboring $\mathrm{Mg}$ atom in the chain, which in turn replaces the second $\mathrm{Mg}$ atom in the 
chain. The third atom is then pushed into a cube-centered interstitial site where it remains stable until the end of the simulation.

The Mg [111] simulation involves the most displacement and replacement events of all the PKAs, as shown by the comparisons in the traces in figure 4(c) and figure 6 , and is therefore the most complicated to describe. The Mg PKA first collides with the nearest neighbor O atom along [111], which is displaced. Temporarily, the Mg PKA resides on the vacant $\mathrm{O}$ site, while additional vacancies are created that will eventually allow it to migrate. The initial $\mathrm{O}$ recoil atom displaces an $\mathrm{Mg}$ atom and then rebounds along [00-1], where it temporarily forms a split interstitial before returning to its original lattice site. The Mg recoil atom that was displaced, with momentum along [111], creates two displacement chains-- one of $\mathrm{Mg}$ and one of $\mathrm{O}$ atoms both along the [110] direction. The $\mathrm{Mg}$ chain along [110] produces two replacement events and terminates with the formation of a $\mathrm{Mg}$ interstitial. Since the $\mathrm{Mg}$ atom that initiated the [110] oriented replacement chains left a vacancy, it provides space for the PKA and adjacent atoms to migrate producing two more replacements. With the $\mathrm{O}$ site vacated by the PKA, its original occupant returns by the end of the simulation. Finally, the [110] aligned O displacement chain produces two replacement events terminating in a split interstitial dumbbell along [110]. In total, six replacement events occurred (4 $\mathrm{Mg}$ and $2 \mathrm{O}$ ) and two Frenkel pairs were formed (1 Mg and $1 \mathrm{O}$ ).

The $\mathrm{O}[100]$ recoil, shown in figure 4(d) creates two split interstitials: one involving the PKA and the other involving an O displaced by the PKA. At first along the [100] direction, the PKA collides with an adjacent $\mathrm{Mg}$ atom. That $\mathrm{Mg}$ recoil atom gains enough kinetic energy to interact with the nearby $\mathrm{O}$ to displace it, at a slight angle, toward the next $\mathrm{Mg}$ atom. Instead of displacing that $\mathrm{Mg}$ atom, the $\mathrm{O}$ recoil deflects slightly and passes through the next three (100) planes. After passing through those three atomic planes, the recoil forms a split interstitial. The PKA rebounds backwards along [100], where it forms a split interstitial, only half a unit cell behind its original lattice site.

The $\mathrm{O}[110]$ recoil has the lowest displacement energy and forms simplest defect configuration of all the cases studied here, as shown in the relatively simple trace in figure 4(e). The PKA forms a split interstitial with the next $\mathrm{O}$ atom along [110]; there are no replacements or other interstitials that form.

Compared to the $\mathrm{Mg}$ [111] recoil event, the $\mathrm{O}$ [111] recoil produces a much simpler defect configurations, as seen by a comparison between the traces in figure 4(c) and figure 4(f). Along the [111] direction, the PKA displaces an $\mathrm{Mg}$ atom that in turn displaces another $\mathrm{O}$ atom. That $\mathrm{Mg}$ atom's trajectory turns to displace another $\mathrm{Mg}$ along [110], but both of these atoms eventually return to their original lattice sites. The $\mathrm{O}$ that had been displaced also forms an unstable split interstitial and then returns to its original lattice site. The PKA rebounds along [010] and forms a split interstitial, which slowly travels over half a unit cell before coming to rest at an interstitial site. 


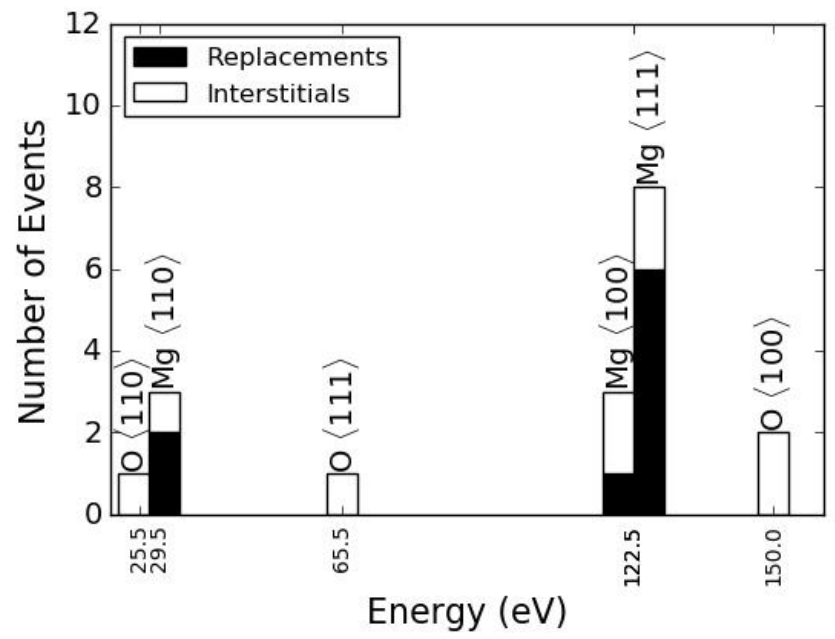

Figure 6: Total number of replacements and interstitial events result in Frenkel pair formation that occurred during each threshold displacement simulation. Interstitial totals include both $\mathrm{Mg}$ and $\mathrm{O}$ atoms.

\section{Discussion}

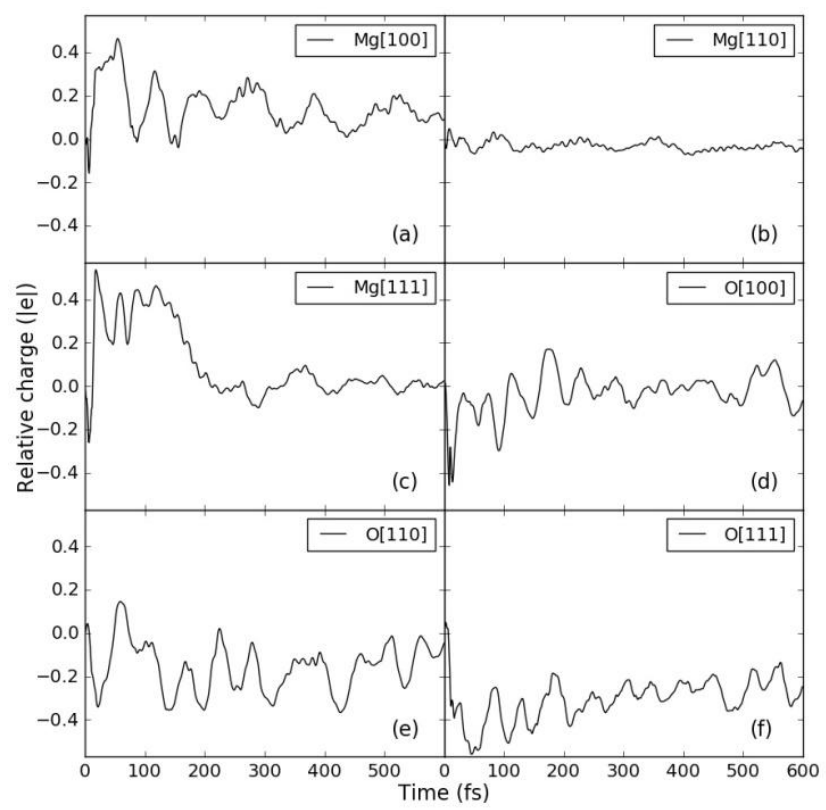

Figure 7: Variation in effective charge of each PKA during the course of the recoil events.

Our results summarized in figure 3 and table 1 show reasonable agreement along a few of the directions with experimental and classical MD values reported in the literature. MD and AIMD show 
higher values for $E_{\mathrm{d}}$ for both $\mathrm{Mg}$ and $\mathrm{O}$ along [100] and [111] directions when compared to experimentally measured values. This is to be expected due to potential sources of experimental uncertainty, such as electron beam misalignment in the case of optical studies and the high dose, high temperature and model dependence in TEM studies of loop formation [6]. Experimentally reported values are all close to $E_{\mathrm{d}}{ }^{\text {ave }}$ of $55 \mathrm{eV}$ [6] regardless of the direction. Along [110], AIMD gives a value about half that of the classical MD and experimental results. Previous studies using classical MD and AIMD have also shown that $E_{\mathrm{d}}$ obtained by AIMD is often lower than that provided by classical MD [38-40]; partialcharge transfer during the collision processes may be the contributing factor to the lower values for $E_{\mathrm{d}}$.

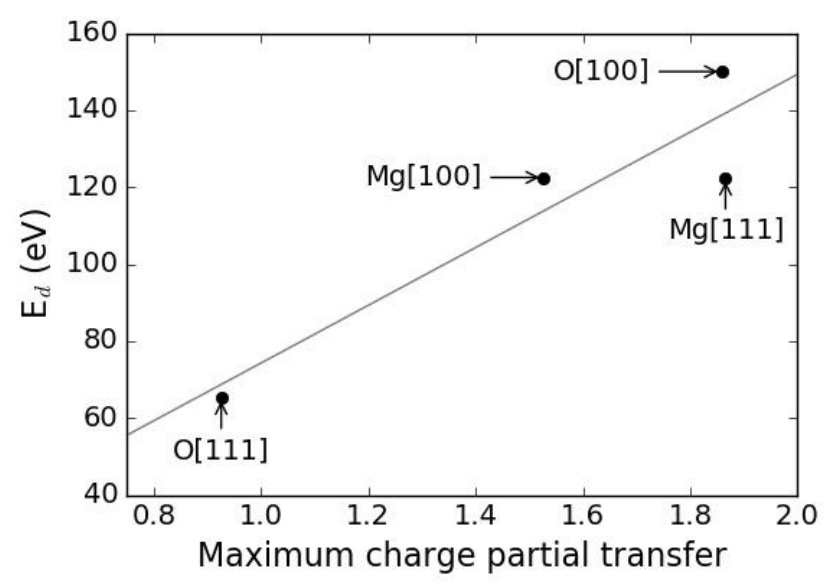

Figure 8: $E_{\mathrm{d}}$ versus partial-charge transfer at the peak potential energy during the simulations. Arrows and labels indicate PKA species and the initial direction of motion. A nearly linear trend between the threshold displacement energies and the amount of partial charge transfer is shown as a gray line to guide the eye.

During each recoil event, the system energy increased as the kinetic energy supplied to the PKA was partially converted to potential energy during the screened-Coulomb interactions. The potential energy may exhibit a peak before coming to rest as a stable configuration. The PKA and subsequent recoil atoms have overlapping orbitals that result in continuous partial-charge transfer. Figure 7 shows the variation in effective charge for each PKA during the simulation. The charge difference is relative to each atom's charge in the initial configuration. The PKAs with the lowest $E_{\mathrm{d}}$ also show the least change in relative charge, especially the $\mathrm{Mg}$ [100] PKA.

The locations of the peaks in system energy reflect the maximum potential energy and most likely represent the maximum screened ion-ion interactions. Figure 8 shows the dependence of $E_{\mathrm{d}}$ on the total amount of partial-charge transfer at the system potential energy peak, $N_{\mathrm{p}}^{\mathrm{ct}}$, which is the difference in the effective Mulliken charge summed over all of the atoms in the system that gain or lose electrons between the initial configuration and the structure at the potential energy peak. Our previous studies have shown a 
nearly linear trend between the threshold displacement energies and the amount of partial charge transfer for $\mathrm{SrTiO}_{3}$, regardless of PKA species [20]. A similar trend is observed for $\mathrm{MgO}$, shown by the trend line in figure 7, which indicates that it might be a universal principle in oxides. Therefore, in such low energy recoil events, the charge redistribution and its effect on the non-equilibrium must be considered. The inability to account for partial-charge transfer in classical MD may be the origin of the discrepancies observed between AIMD and classical MD results. Deficiencies in classical MD potentials may also play a role, and results, such as presented here, may provide benchmarks for refining classical potentials in simple and complex oxides. Charge transfer potentials are another possible way to improve classical MD to yield results comparable to those of AIMD [41-43].

\section{Conclusion}

Low-energy recoil events in $\mathrm{MgO}$ are investigated using ab initio molecular dynamics simulations, based on density functional theory. The threshold displacement energies are shown to be highly directional dependent; displacements along single-species atomic chains occurred far more easily than those along mixed-species atomic chains. The two minimum $E_{\mathrm{d}}$ values $(25.5 \mathrm{eV}$ for $\mathrm{O}$ and $29.5 \mathrm{eV}$ for $\mathrm{Mg}$ ) both occur along the [110] single-species atomic chains. Low-energy recoil events of $\mathrm{Mg}$ and $\mathrm{O}$ form two types of interstitials; while $\mathrm{Mg}$ prefers to sit on cube centered sites, O prefers to form [110] aligned split interstitials. Compared to classical MD simulations, AIMD follows the same general trends. The lower $E_{\mathrm{d}}$ values from AIMD calculations are attributed to partial-charge transfer assisted processes. Charge transfer potentials in classical MD may be a possible approach to better predict defect dynamics under irradiation. Since $\mathrm{MgO}$ is used as a model material due to its simple electronic and atomic structure, these results are useful when considering radiation effects in ceramic oxides and in particular the radiation resistance of crystals with the cubic crystal structure.

\section{Acknowledgements}

This work was supported by the U.S. Department of Energy, Office of Science, Basic Energy Sciences, Materials Sciences and Engineering Division. One of the authors (BAP) was supported by the U.S. Department of Energy, Office of Nuclear Energy, Nuclear Energy University Program. The theoretical calculations were performed using the supercomputer resources at the National Energy Research Scientific Computing Center, supported by the Office of Science, US Department of Energy under Contract No.DEAC02-05CH11231.

\section{References}

1. Weber WJ, Ewing RC, Catlow CRA, de la Rubia TD, Hobbs LW, Kinoshita C, et al. Radiation effects in crystalline ceramics for the immobilization of high-level nuclear waste and plutonium. $\mathrm{J}$ Mater Res. 1998;13(06):1434-84. 
2. Kinoshita C, Zinkle SJ. Potential and limitations of ceramics in terms of structural and electrical integrity in fusion environments. J Nucl Mater. 1996 Oct 1;233-237, Part 1:100-10.

3. Moll S, Zhang Y, Debelle A, Thome L, Crocombette JP, Zihua Z, et al. Damage processes in MgO irradiated with medium-energy heavy ions. Acta Mater. 2015 Apr 15;88:314-22.

4. Degueldre C, Yamashita T. Inert matrix fuel strategies in the nuclear fuel cycle: the status of the initiative efforts at the 8th Inert Matrix Fuel Workshop. J Nucl Mater. 2003 Jun 1;319:1-5.

5. Sickafus KE, Kotomin EA, Uberuaga BP. Radiation Effects in Solids. Springer Science \& Business Media; 2007. 593 p.

6. Zinkle SJ, Kinoshita C. Defect production in ceramics. J Nucl Mater. 1997 Nov;251:200-17.

7. Ziegler JF, Ziegler MD, Biersack JP. SRIM - The stopping and range of ions in matter (2010). Nucl Instrum Methods Phys Res B. 2010 Jun 1;268:1818-23.

8. Nordlund K, Djurabekova F. Multiscale modelling of irradiation in nanostructures. J Comput Electron. 2014 Mar;13(1):122-41.

9. Thomas BS, Marks NA, Begg BD. Defects and threshold displacement energies in $\operatorname{SrTiO}(3)$ perovskite using atomistic computer simulations. Nucl Instrum Methods Phys Res Sect B-Beam Interact Mater At. 2007 Jan;254(2):211-8.

10. Bacon DJ, Gao F, Osetsky YN. The primary damage state in fcc, bcc and hcp metals as seen in molecular dynamics simulations. J Nucl Mater. 2000 Jan 1;276:1-12.

11. Gao F, Weber WJ. Cascade overlap and amorphization in 3C-SiC: Defect accumulation, topological features, and disordering. Phys Rev B. 2002 Jul 1;66(2):024106.

12. Pedro A. F.P. Moreira RD. Molecular-dynamics simulation of threshold displacement energies in zircon. Nucl Instrum Methods Phys Res Sect B Beam Interact Mater At. 2009;267(20):3431-6.

13. Mulroue J, Duffy DM. An ab initio study of the effect of charge localization on oxygen defect formation and migration energies in magnesium oxide. Proc R Soc -Math Phys Eng Sci. 2011 Jul 8;467(2131):2054-65.

14. Gao F, Xiao H, Zu X, Posselt M, Weber WJ. Defect-Enhanced Charge Transfer by Ion-Solid Interactions in SiC using Large-Scale Ab Initio Molecular Dynamics Simulations. Phys Rev Lett. 2009 Jul 10;103(2):027405.

15. Soler JM, Artacho E, Gale JD, Garcia A, Junquera J, Ordejon P, et al. The SIESTA method for ab initio order-N materials simulation. J Phys-Condens Matter. 2002 Mar 25;14(11):2745-79.

16. Troullier N, Martins J. Efficient Pseudopotentials for Plane-Wave Calculations. Phys Rev B. 1991 Jan 15;43(3):1993-2006.

17. Kleinman L, Bylander D. Efficacious Form for Model Pseudopotentials. Phys Rev Lett. 1982;48(20):1425-8.

18. Perdew JP, Burke K, Ernzerhof M. Generalized gradient approximation made simple. Phys Rev Lett. 1996 Oct 28;77(18):3865-8. 
19. Liu B, Xiao HY, Zhang Y, Weber WJ. Ab initio molecular dynamics simulations of overlapping recoil events in ThO2. J Phys-Condens Matter. 2013 Oct 2;25(39):395004.

20. Liu B, Xiao HY, Zhang Y, Aidhy DS, Weber WJ. Ab initio molecular dynamics simulations of threshold displacement energies in SrTiO3. J Phys-Condens Matter. 2013 Dec 4;25(48):485003.

21. Holmström E, Kuronen A, Nordlund K. Threshold defect production in silicon determined by density functional theory molecular dynamics simulations. Phys Rev B. 2008 Jul 9;78(4):045202.

22. Hazen R. Effects of Temperature and Pressure on Cell Dimension and X-Ray Temperature Factors of Periclase. Am Mineral. 1976;61(3-4):266-71.

23. Sasaki S, Fujino K, Takeuchi Y. X-Ray Determination of Electron-Density Distributions in Oxides, Mgo, Mno, C0o, and Nio, and Atomic Scattering Factors of Their Constituent Atoms. Proc Jpn Acad Ser B-Phys Biol Sci. 1979;55(2):43-8.

24. Tsirelson VG, Avilov AS, Abramov YA, Belokoneva EL, Kitaneh R, Feil D. X-ray and electron diffraction study of MgO. Acta Crystallogr Sect B-Struct Sci. 1998 Feb 1;54:8-17.

25. Karen P, Kjekshus A, Huang Q, Karen VL. The crystal structure of magnesium dicarbide. J Alloys Compd. 1999 Jan 15;282(1-2):72-5.

26. Boiocchi M, Caucia F, Merli M, Prella D, Ungaretti L. Crystal-chemical reasons for the immiscibility of periclase and wustite under lithospheric P, T conditions. Eur J Mineral. 2001 Oct;13(5):871-81.

27. Isaak D, Anderson O, Goto T. Measured Elastic-Moduli of Single-Crystal Mgo up to 1800-K. Phys Chem Miner. 1989;16(7):704-13.

28. Anderson O, Andreatc.p. Pressure Derivatives of Elastic Constants of Single-Crystal Mgo at 23 Degrees and -195.8 Degrees C. J Am Ceram Soc. 1966;49(8):404 - \&.

29. Mao H, Bell P. Equations of State of Mgo and Epsilon-Fe Under Static Pressure Conditions. J Geophys Res. 1979;84(NB9):4533-6.

30. Mehl M, Cohen R, Krakauer H. Linearized Augmented Plane-Wave Electronic-Structure Calculations for Mgo and Cao. J Geophys Res-Solid Earth Planets. 1988 Jul 10;93(B7):8009 - +.

31. Jaffe JE, Snyder JA, Lin ZJ, Hess AC. LDA and GGA calculations for high-pressure phase transitions in $\mathrm{ZnO}$ and MgO. Phys Rev B. 2000 Jul 15;62(3):1660-5.

32. Pells G. The Temperature-Dependence of the Threshold Displacement Energy in Mgo. Radiat Eff Defects Solids. 1982;64(1-4):71-5.

33. Park B, Weber WJ, Corrales LR. Molecular dynamics study of the threshold displacement energy in MgO. Nucl Instrum Methods Phys Res Sect B-Beam Interact Mater At. 2000 May;166:357-63.

34. Kittiratanawasin L, Smith R, Uberuaga BP, Sickafus K. Displacement threshold and Frenkel pair formation energy in ionic systems. Nucl Instrum Methods Phys Res Sect B-Beam Interact Mater At. 2010 Oct 1;268(19):2901-6. 
35. Sonoda T, Kinoshita C, Isobe Y. Static and dynamic properties of point defects in magnesia. Ann Phys. 1995 Jun;20(3):33-43.

36. Brudevoll T, Kotomin EA, Christensen NE. Interstitial-oxygen-atom diffusion in MgO. Phys Rev B. 1996 Mar 15;53(12):7731-5.

37. Uberuaga BP, Smith R, Cleave AR, Henkelman G, Grimes RW, Voter AF, et al. Dynamical simulations of radiation damage and defect mobility in MgO. Phys Rev B. 2005 Mar 3;71(10):104102.

38. Xiao HY, Gao F, Zu XT, Weber WJ. Threshold displacement energy in GaN: Ab initio molecular dynamics study. J Appl Phys. 2009 Jun 15;105(12):123527.

39. Xiao HY, Gao F, Weber WJ. Threshold displacement energies and defect formation energies in Y2Ti2O7. J Phys-Condens Matter. 2010 Oct 20;22(41):415801.

40. Wang XJ, Xiao HY, Zu XT, Zhang Y, Weber WJ. Ab initio molecular dynamics simulations of ionsolid interactions in Gd2Zr2O7 and Gd2Ti2O7. J Mater Chem C. 2013;1(8):1665-73.

41. Weber WJ, Zarkadoula E, Pakarinen OH, Sachan R, Chisholm MF, Liu P, et al. Synergy of elastic and inelastic energy loss on ion track formation in SrTiO3. Sci Rep. 2015 Jan 12;5:7726.

42. Zhang Y, Sachan R, Pakarinen OH, Chisholm MF, Liu P, Xue H, et al. Ionization-induced annealing of pre-existing defects in silicon carbide. Nat Commun. 2015 Aug 12;6:8049.

43. Weber WJ, Duffy DM, Thomé L, Zhang Y. The role of electronic energy loss in ion beam modification of materials. Curr Opin Solid State Mater Sci. 2015 Feb;19(1):1-11. 